Evaluar.

2004, $n^{\circ} 4$ (setiembre)
Laboratorio de Evaluación Psicológica y Educativa. Facultad de Psicología Universidad Nacional de Córdoba (Argentina). ISSN N ${ }^{\circ} 1515$ - 1867

\title{
Adaptación del Inventario de Personalidad 16PF-IPIP a un Contexto de Orientación. Estudio Preliminar
}

\author{
Edgardo Pérez* ${ }^{1}$, Marcos Cupani* \& Carlos Beltramino** \\ * Lab. de Evaluación Psicológica y Educativa. Facultad de Psicología. Universidad Nacional de Córdoba. \\ ** Instituto de Investigaciones Médicas Mercedes y Martín Ferreyra
}

\begin{abstract}
Resumen: En nuestra región se han creado varios instrumentos para fines de orientación de carrera: El Cuestionario de Intereses Profesionales Revisado, el Inventario de Autoeficacia para Inteligencias Múltiples y el Sistema de Orientación Vocacional Informatizado (Fogliatto \& Pérez, 2003). Sin embargo, no se dispone de escalas de medición de rasgos de personalidad adecuadas para un contexto de orientación. Investigaciones recientes (Tokar, Fisher \& Subich, 1998) han demostrado que este tipo de medidas mejoran la predicción de variables con implicancia vocacional, tales como elección de carrera, rendimiento académico y satisfacción con el empleo. Hemos comenzado a adaptar un instrumento promisorio: el 16PF en la versión del Pool Internacional de Items de Personalidad (IPIP, por sus siglas en inglés) del Dr. Lewis Goldberg. Hasta el momento se ha realizado la traducción inversa del inventario, la verificación de la consistencia interna de sus escalas y un estudio de validez de criterio respecto a la variable intenciones de elección de carrera, todos con resultados satisfactorios. Actualmente se están planificando estudios de validez incremental y comparativa del 16PF IPIP con otras medidas empleadas en orientación para la carrera vocacional (habilidades objetivas, autoeficacia e intereses).
\end{abstract}

Palabras Clave: Teoría de los Cinco Factores- Inventario IPIP- 16 PF- Orientación para la carrera.

\section{Introducción}

La construcción y adaptación de instrumentos locales de medición utilizados en un proceso de Orientación Vocacional fue creciendo paulatinamente desde que se diseñó el

\footnotetext{
${ }^{1}$ La correspondencia relacionada con este artículo debe ser dirigida a Edgardo Pérez. David Luque 134. Piso 2, Dpto F. CP: 5000.E-mail: edrape@onenet.com.ar
} 
Cuestionario de Intereses Profesionales (Fogliatto, 1991) hasta la presentación de un Sistema de Orientación Vocacional Informatizado, en su tercera versión (SOVI 3), que contempla información sobre carreras y especialidades del Polimodal, así como evaluación de autoeficacia para inteligencias múltiples e intereses vocacionales (Fogliatto y Pérez, 2003). No contamos, por el momento, con un inventario de personalidad verdaderamente adaptado a las características de nuestra población (Pérez \& Baldo, 2002).

En Orientación para la carrera ha resurgido el interés por la medición de la personalidad. En efecto, varias investigaciones intentan vincular modelos teóricos de la personalidad con otros de los intereses vocacionales (particularmente la tipología de Holland, 1997) y determinar el papel de la evaluación de la personalidad en orientación para la carrera (Lowman, 1991, Gottfreson, Jones \& Holland, 1993). Larson, Rottinghaus \& Borgen (2002) realizaron un meta-análisis que obtuvo evidencias de las relaciones entre las medidas más usadas para evaluar los intereses (el Self Directed Search, por ejemplo) y la medida más popular para evaluar los cincos factores, el NEO Personality Inventory (Costa \& McCrae, 1999). De las treintas correlaciones observadas, cincos parecen ser sustanciales tanto para hombres y mujeres, como para todas las medidas de intereses. Otras investigaciones (Tokar, Fisher \& Subich,1998) han demostrado que la evaluación de la personalidad es significativamente predictiva de comportamientos de elección de carrera, de variables relacionadas con las carreras (tales como intereses y valores) y variables relacionadas con la adaptación a las carreras (satisfacción ocupacional, por ejemplo).

El modelo de mayor consenso para la descripción de los rasgos es el de los cinco grandes factores (Five-Factor Model, McCrae \& Costa, 1992) que parece representar un avance conceptual y empírico en el campo de la teoría de la personalidad. La teoría de los cinco factores (FFM) es un modelo conceptual que condensa décadas de análisis factorial llevado a cabo con muestras de personas de distintas edades y sexo, en diferentes culturas y un extenso número de autoinformes e informes de pares sobre adjetivos descriptivos de la personalidad e ítems de cuestionarios (Costa \& McCrae, 1999). Como resultado de esta larga serie de investigaciones se han obtenido cinco factores bipolares (Goldberg, 1992; John, 1990) que fueron denominados como: Factor I, Extraversión (reserva vs animación); Factor II, Amabilidad (hostil vs empático); 
Factor III, Responsabilidad (bien organizado vs. impulsivo); Factor IV, Neuroticismo (inestable vs. estable emocionalmente); y Factor V, Cultura (imaginativo vs concreto). Este último factor también ha sido interpretado como Intelecto (Digman \& TakemotoChock, 1981) o como Apertura a la Experiencia (McCrae \& Costa, 1999).

Este modelo supone que los rasgos más importantes de la personalidad estarían codificados en el lenguaje natural y que los individuos en el transcurso de la historia, han utilizado adjetivos que denotan rasgos para poder describirse o describir a otras personas. Francis Galton (1884) es el precursor de la hipótesis léxica, donde "las diferencias más importantes en las transacciones humanas están codificadas como términos simples en algunos o todos los lenguajes del mundo" (Goldberg, 1993). El trabajo de Galton fue continuado por Allport y Olbert (1936) que propusieron una lista exhaustiva de 4.500 adjetivos relevantes; y más tarde, este trabajo sería replicado por Norman (1963).

Raymond B. Cattell (1947) emprendió el primer programa científico relevante en este ámbito. Cattell se propuso reducir la mayor cantidad de adjetivos para obtener un conjunto de categorías optimas, que condensara la mayor cantidad de información de la lista de adjetivos de personalidad. Con el método de análisis factorial, pudo reducir miles de descriptores a 16 factores primarios de personalidad, que sirvieron para construir el Inventario de Personalidad 16PF (Sixteen Personality Factor Inventory). No obstante, varios especialistas realizaron análisis factorial de segundo orden con los 16 factores primarios, encontrando cinco factores recurrentes y altamente confiables (Norman, 1963; Tupes \& Cristal, 1961, 1992).

Sin embargo, no todos los investigadores de la teoría de los rasgos de personalidad están de acuerdo con el FFM (Eysenck, 1992; Block, 1995, 2001). MacAdams (1992) resume las críticas que se han formulado al mismo. Entre ellas se destacan: a) falta de verdadera independencia entre los factores, puesto que si bien los mismos son descriptos como ortogonales o casi ortogonales (Goldberg, 1992), los resultados empíricos indican que frecuentemente los cinco factores están intercorrelacionados; b) limitaciones del análisis factorial, puesto que la recurrencia de la estructura factorial puede ser atribuible a la similitud de las variables empleadas más que a la estructura intrínseca de la personalidad (Block, 1995), y c) carencia de una teoría biológica o psicológica de la personalidad, puesto que el FFM no se desarrolla a 
partir de una teoría explícita, si bien luego se han construido modelos explicativos adhoc que intentan vincularlo con mecanismos biológicos (Costa \& McCrae, 1999).

Pese a las críticas enunciadas, la mayoría de los investigadores coinciden en que ahora disponemos de un marco general para una estructura comprensiva de los atributos fenotípicos de la personalidad (Digman, 1990; John, 1990, Saucier \& Goldberg, 1995). La contribución de los estudios transculturales (McCrae, 2003), genéticos (Loehlin et al,1998) y la aplicación de técnicas de neuroimagen (Johnson et al.,1999) han permitido un sistemático avance en la comprensión de la estructura básica de la personalidad.

Investigaciones realizadas (McCrae, 2003) sobre una variedad de lenguas indoeuropeas y otras, recolectando una extensa muestra de adjetivos descriptivos de rasgos, conducen generalmente a la representación de los cinco grandes factores, la que permite comprender la mayoría de los atributos fenotípicos de personalidad (Goldberg, 1993). El hecho que los FFM se han encontrado en distintas culturas sugiere que las características básicas de la personalidad que dan origen a los factores tienen una base biológica.

Los estudios realizados en Estados Unidos (Waller, 1999), Canadá (Jang, Livesley, and Veron, 1996), y Alemania (Riemann et al., 1997) aplicando modelos estructurales, han demostrado que la herencia genética oscila en un rango de .48 a .60 de la varianza, lo que demuestra que los cinco factores estarían influenciados, al menos parcialmente, por mecanismos biológicos. Algunos especialistas, desde un enfoque evolutivo-filogenético, especulan, que los rasgos de personalidad serían transmitidos de generación en generación como resultado de estrategias de afrontamiento necesarias para la supervivencia y el desarrollo (Bouchard \& Loehlin, 2001).

Las investigaciones realizadas con técnicas de neuroimagen funcional, también han aportado evidencias empíricas interesantes. Johnson et al. (1999) utilizando Tomografía por Emisión de Positrones (PET) encontró una correlación significativa entre los sujetos introvertidos y la actividad cerebral en el lóbulo frontal y tálamo anterior, mientras que los sujetos extrovertidos (E), presentaban un incremento de actividad cerebral en el lóbulo temporal, tálamo posterior y el giro cingular anterior.

En la literatura especializada, puede observarse que los factores Extraversión y Neuroticismo son los que mayor evidencia presentan, ya que desde la teoría de los rasgos, son compartidos por otros modelos taxonómicos, como el modelo PEN de 
Eysenck (1991), por ejemplo. Sin embargo, en los últimos años se han realizados nuevas investigaciones con el modelo FFM, especialmente con los factores Apertura (O), Amabilidad (A) y Responsabilidad (R). En un estudio exploratorio (Stuogh, et. al., 2001) utilizando electroencefalograma (EEG) y el inventario NEO PI-R, se reportaron correlaciones significativas entre los factores $\mathrm{O}$, A y R con los patrones de actividad neuronal (theta, alfa, beta-1 y beta-2) en diferentes bandas del EEG localizadas en el lóbulo frontal, el cortex occipital-parietal y el área central-temporal.

Es necesario seguir investigando la relación entre la base genotípica de la personalidad y los mecanismos funcionales del cerebro (Bouchard \& Loehlin, 2001), puesto, que tal como argumentó Cloninger (1988), ninguna dimensión de la personalidad puede ser tomada seriamente a menos que sea apoyada por una teoría que la vincule a mecanismos biológicos subyacentes.

El FFM no es privativo de un instrumento en particular, en realidad es una descripción y explicación de la personalidad que posee diversos inventarios construidos para su evaluación. Dentro de los instrumentos mas reconocidos e investigados están el Sixteen Personality Factor Inventory (16PF-5) (Russelll \& Karol, 2000) y el NEO PI-R y su versión abreviada NEO-FFI (Costa \& McCrae, 1999). En estos últimos años se destaca el esfuerzo de Goldberg (1999) por generar un banco on-line de ítem de personalidad de dominio público (IPIP, por sus siglas en inglés), con el propósito de fomentar nuevas investigaciones relacionadas con la aplicación, adaptación y construcción de escalas FFM en todo el mundo. Una extensa investigación comparativa entre inventarios de personalidad dirigida por Goldberg (2001) ha demostrado que el $16 \mathrm{PF}$, versión IPIP, posee las mejores propiedades psicométricas y superior validez criterial.

Por consiguiente, nos hemos propuesto traducir y adaptar el Inventario de Personalidad 16PF-IPIP de Goldberg (1999), realizando los siguientes estudios específicos: traducir el instrumento; verificar la consistencia interna de cada una de sus escalas; y analizar la validez de criterio de las escalas con respecto a la variable Intenciones de elección de carrera. De este modo se cree brindar una contribución significativa al instrumental evaluativo empleado por los orientadores vocacionales de nuestra región. 
No son muchas las investigaciones que se han ocupado de las relaciones entre las medidas del FFM y elección de carrera. Lapan, Shughnessy \& Boggs (1996) informaron acerca de una modesta pero significativa relación inversa entre Extraversión y elección de una carrera científica-matemática, empleando el NEO-PI-R, en una muestra de estudiantes ingresantes a la universidad. Los estudios de validación del 16PF (Kline, 2000) también comprobaron perfiles de personalidad diferenciados entre numerosos grupos ocupacionales. Estos descubrimientos son consistentes con otras evidencias relacionadas con diferencias entre orientación a personas y orientación técnica en las especialidades médicas (Borges \& Osmon, 2000). Moreno et al (2002) analizaron la relación entre determinados rasgos de personalidad y la profesión médica o sus distintas especialidades, aplicando el 16PF original de Cattell. El inventario 16PF fue administrado a 161 médicos (de familia, anestesistas y cirujanos) y se observaron diferencias medias significativas entre los grupos de especialistas en tres factores primarios (atención a normas, abstracción y vigilancia) y uno de segundo orden (Apertura). Un análisis discriminante demostró que, entre los 16 factores primarios, atención a normas y abstracción evidenciaron ser los rasgos que mejor discriminaron entre los grupos de especialistas y, que, entre los factores globales, apertura fue el constructo de mayor poder discriminante. Así, los estudiantes que habían optado por las especialidades de "Pediatría", "Ginecología" y "especialidades sensoriales" tendieron a ser respecto al resto de estudiantes más "afectuosos" (A+), “confiados" (L-) y "prácticos" (M-); los que escogieron la especialidad de "Psiquiatría" fueron más "sensibles" (I+) e "imaginativos" (M+) y los que prefirieron "Traumatología" “duros"(I-) y "prácticos"(M-). Los que optaron por las especialidades de "Medicina Interna" y "Cirugía" no presentaron un perfil de personalidad diferenciado del conjunto de estudiantes de Medicina.

Goldberg (1999) en un estudio comparativo de la validez de cinco inventarios de Personalidad para predecir 6 grupos (Clusters) de frecuencia de actividades cotidianas en una muestra de 423 adultos, descubrió que las escalas del 16PF-IPIP (su versión del famoso Inventario) eran las de mayor valor predictivo, utilizando análisis de regresión múltiple con el procedimiento Stepwise. De este modo, atención a normas (una faceta de autocontrol-responsabilidad) fue el mejor predictor de la frecuencia del consumo de drogas (peso estandarizado -.35) y comportamiento informal (-.32) y facetas del factor 
Amabilidad y Apertura (sensibilidad, complejidad e intelecto, respectivamente) de actividades de lectura (.49), escritura (.29) y logros creativos (.26)

\section{Método}

\section{Instrumento}

El Inventario de 16 factores de personalidad, versión IPIP (16PF-IPIP), comprende 163 ítems construidos para medir las 16 escalas primarias y cincos dimensiones globales de la personalidad en adolescentes y adultos sin trastornos psicológicos severos (Ver Apéndice). Cada ítem esta redactado en forma de frase que describe comportamientos típicos de las personas y se solicita al sujeto que evalúe el grado de precisión con que cada oración lo describe, utilizando una escala de cinco opciones de respuestas (Muy en desacuerdo con esta descripción de mi mismo, En desacuerdo, Ni de acuerdo ni en desacuerdo, De acuerdo y Muy de acuerdo).

El Cuestionario de Intenciones de Elección de Carrera (CIEC, Pérez, 2001) es un listado de 42 carreras de nivel Universitario y Terciario, que conforman seis escalas obtenidas por análisis factorial (Carreras Sociales, Tecnológicas, Médicas, Artísticas, Humanistas y Científicas). En este cuestionario se le solicita al individuo que evalúe en una escala de 1 a 10 sus probabilidades de inscribirse en cada carrera del listado (Psicología, por ejemplo). Las opciones de repuestas van de 1 - "Es muy improbable que me inscriba en esa carrera" a 10 - "Es muy probable que me inscriba en esa carrera”. Las seis escalas poseen valores de consistencia interna adecuados (alfa .74 a $.90)$, en especial si se considera el reducido número de ítems de algunas de ellas.

\section{Muestra}

Para cada uno de los estudios realizados se describe a continuación la muestra de investigación empleada. Se procuró que las muestras fueran lo más heterogéneas posibles en relación con sexo, nivel económico y orientación del polimodal.

\section{Estudio 1. Traducción del 16PF-IPIP}

La adaptación de un test es un proceso complejo que implica considerar no solo la mera traducción sino también las variables culturales involucradas para controlar las fuentes de sesgos. En primer lugar se aplicó el método de traducción inversa (back translation), 
donde el test fue traducido del inglés al español por uno de nosotros y, posteriormente, especialistas americanos que dominaban también el idioma español procedieron a traducir esta versión española al idioma inglés. Esta traducción fue comparada luego con la versión original en inglés y se realizaron los ajustes necesarios. Para este procedimiento se contó con el asesoramiento del Dr. Richard Póstuma (Universidad de El Paso, Texas), un colaborador del Dr. Lewis Goldberg.

En segundo lugar, tanto la versión del inventario en inglés como en español fueron aplicadas a una muestra bilingüe de 49 estudiantes (varones 10 y mujeres 39; media de edad: 24,5) de la ciudad de Córdoba, Argentina, que cursaban el último año del Traductorado de Inglés en la Facultad de Lengua de la Universidad Nacional de Córdoba. Se administró el test en su versión en Español y, dos semanas después, se aplicó la prueba en inglés. Las puntuaciones obtenidas en las escalas semejantes de ambas versiones del inventario (Calidez en español vs Warmth en inglés, por ejemplo) se correlacionaron empleando el coeficiente $\mathrm{r}$ de Pearson y, posteriormente, se obtuvo el coeficiente de correlación corregido por atenuación.

Este último parámetro se utiliza para estimar el valor verdadero de la correlación entre variables, atenuando el error de medición de las mismas (Nunnally \& Bernstein, 1995), y se recomienda cuando hay situaciones de evaluación que hacen sospechar un déficit de la confiabilidad de las medidas empleadas, como es el caso de una muestra pequeña de investigación. En la tabla siguiente se presentan los coeficientes de correlación entre las escalas equivalentes de ambas versiones del 16PF-IPIP (español e inglés) y el coeficiente de confiabilidad corregido por atenuación para las 16 escalas de las dos versiones del inventario. 


\section{Tabla 1}

Coeficientes de correlación y coeficientes de confiabilidad corregido por atenuación entre las escalas de las dos versiones del inventario (español e inglés).

\begin{tabular}{|c|c|c|}
\hline Escalas & $\mathbf{r}$ & $\begin{array}{c}\text { r x-y } \\
\text { corrección }\end{array}$ \\
\hline Calidez & .80 & 1.08 \\
\hline Intelecto & .64 & .96 \\
\hline Estabilidad & .84 & 1.07 \\
\hline Asertividad & .74 & .94 \\
\hline Gregarismo & .85 & 1.02 \\
\hline Obediencia & .87 & 1.07 \\
\hline Amigabilidad & .86 & .95 \\
\hline Sensibilidad & .86 & 1.23 \\
\hline Confianza & .72 & .95 \\
\hline Imaginación & .83 & 1.06 \\
\hline Apertura & .81 & .87 \\
\hline Autoestima & .71 & .95 \\
\hline Complejidad & .82 & .98 \\
\hline Sociabilidad & .80 & 1.17 \\
\hline Perfeccionismo & .80 & 1.04 \\
\hline Calma & .84 & 1.07 \\
\hline
\end{tabular}

Como puede observarse, los coeficientes de correlación entre las 16 escalas de las dos versiones del 16PF-IPIP son elevados y, por consiguiente, puede afirmarse que las escalas son equivalentes en términos generales.

\section{Estudio 2. Consistencia Interna}

Se administró el Inventario 16PF-IPIP a una muestra de 418 adolescentes de ambos sexos $(67,7 \%$ mujeres; $32.3 \%$ varones; media de edad: 17,29) que cursaban el ciclo educativo Polimodal en sus diversas orientaciones. Los datos fueron ingresados a una base de datos del paquete estadístico SPSS 11.5 y se verificó la consistencia interna de las 16 escalas primarias mediante el coeficiente alfa. Este procedimiento es el más empleado en la actualidad para corroborar esta dimensión de la confiabilidad y es un indicador de la co-variación de los ítems de una escala (Muñiz, 2001). En la tabla 2 se presentan los coeficientes alfa de las 16 escalas del inventario 16PF IPIP y los de la versión original del instrumento en inglés. 
Tabla 2

Coeficiente alfa de las 16 escalas del inventario 16PF IPIP, versiones en español e inglés

\begin{tabular}{llcc}
\hline \multirow{2}{*}{ Factor } & \multicolumn{1}{c}{ Escalas } & \multicolumn{2}{c}{$\alpha$ de Cronbach } \\
& Amigabilidad & .85 & .80 \\
Expañol & Ingles \\
\hline \multirow{3}{*}{ Extraversión } & Apertura & .89 & .86 \\
& Asertividad & .75 & .81 \\
& Gregarismo & .79 & .78 \\
Amabilidad & Sociabilidad & .76 & .73 \\
Responsabilidad & Calidez & .63 & .80 \\
& Sensibilidad & .72 & .73 \\
& Perfeccionismo & .82 & .81 \\
Estabilidad & Obediencia & .73 & .83 \\
Emocional & Estabilidad & .83 & .85 \\
& Autoestima & .80 & .80 \\
\multirow{3}{*}{ Cultura } & Calma & .79 & .76 \\
& Confianza & .77 & .80 \\
Coeficiente $\alpha$ & Complejidad & .78 & .82 \\
promedio & Intelecto & .72 & .76 \\
\hline
\end{tabular}

Como puede apreciarse la consistencia interna es adecuada para prácticamente todas las escalas de la versión en español (ligeramente insatisfactoria en la escala Calidez), con valores muy semejantes a los de la versión en idioma inglés de este inventario (Goldberg, 1999).

\section{Estudio 3. Evidencia de validez de criterio}

Para el estudio de validez de criterio respecto a la variable Intenciones de Elección de Carreras se utilizó el Cuestionario de Intenciones de Elección de Carreras (CIEC, Pérez, 2001).

Se administró el 16PF-IPIP a una muestra de 240 adolescentes $(64,4 \%$ mujeres; 35,6 \% varones; media de edad: 17,22) que cursaban el ciclo educativo Polimodal en sus diversas orientaciones y, tres meses después, al finalizar el año electivo, los mismos estudiantes fueron evaluados con el CIEC. En primer lugar se correlacionaron las puntuaciones obtenidas en los cinco factores del 16PF IPIP versus los seis factores del CIEC y, posteriormente, se correlacionaron estos últimos con las 16 escalas primarias 
del 16PF-IPIP. En general, la mayoría de los investigadores consideran que es mas importante para fines aplicados el conocimiento de las facetas específicas que el meramente obtenido de los cincos grandes factores (Goldberg, 1999). En las tablas 3 y 4 se presentan estos resultados.

Tabla 3

Correlaciones entre los Cinco Factores y las seis escalas del CIEC.

\begin{tabular}{lcccccc}
\hline \multirow{2}{*}{$\begin{array}{c}\text { Cinco } \\
\text { Factores }\end{array}$} & $\begin{array}{c}\text { Carreras } \\
\text { Médicas }\end{array}$ & $\begin{array}{c}\text { Carreras } \\
\text { Tecnológicas }\end{array}$ & $\begin{array}{c}\text { Carreras } \\
\text { Sociales }\end{array}$ & $\begin{array}{c}\text { Carreras } \\
\text { Humanistas }\end{array}$ & $\begin{array}{c}\text { Carreras } \\
\text { Artísticas }\end{array}$ & $\begin{array}{c}\text { Carreras } \\
\text { Científicas }\end{array}$ \\
\hline Extraversión & .000 & -.005 & $.149^{*}$ & .038 & .081 &.-.015 \\
Amabilidad & .057 & $-.372^{* *}$ & .097 & $.352^{* *}$ & $.256^{* *}$ & $-.200^{* *}$ \\
Responsabilidad & .125 & -.071 & .041 &, 077 & $-.167^{* *}$ & -.014 \\
Estabilidad & .018 & $.163 *$ & .035 & -.053 & -.098 & -.082 \\
Emocional & & & & & & \\
Cultura & -.060 & -.127 & .015 & $.247^{* *}$ & $.242^{* *}$ & -.043 \\
\hline$* \mathrm{p}<.05$ & $* * \mathrm{p}<.01$ & & & & &
\end{tabular}


Tabla 4

Coeficientes de correlación entre las 16 escalas primarias del 16PF-IPIP y las seis escalas del CIEC.

\begin{tabular}{lcccccc}
\hline \multirow{2}{*}{ Facetas } & \multicolumn{5}{c}{ Escalas del CIEC } \\
& Carreras & Carreras & Carreras & Carreras & Carreras & Carreras \\
& Médicas & Tecnológicas & Sociales & Humanistas & Artísticas & Científicas \\
\hline Calidez & -.008 & -.091 & $.189^{* *}$ & $.200^{* *}$ & $.140^{*}$ & -.064 \\
Intelecto & -.017 & -.028 & .007 & .102 & -.011 & -.041 \\
Estabilidad & .008 & $.231^{* *}$ & .051 & -.092 & $-.156^{*}$ & .084 \\
Asertividad & -.034 & .007 & .057 & -.017 & -.069 & .028 \\
Gregarismo & -.001 & $.171^{* *}$ & $.173^{* *}$ & .065 & $.160^{*}$ & .120 \\
Obediencia & $.170^{* *}$ & -.014 & .070 & .041 & $-.191^{*}$ & .027 \\
Amigabilidad & -.037 & -.041 & $.152^{* *}$ & .057 & $.143^{*}$ & -.059 \\
Sensibilidad & .078 & $-.426^{* *}$ & .000 & $.326^{* *}$ & $.242^{* *}$ & $-.221 * *$ \\
Confianza & .038 & -.018 & .016 & .078 & .004 & -.003 \\
Imaginación & -.057 & -.068 & .013 & .104 & $.250^{* *}$ & -.022 \\
Apertura & -.005 & -.095 & .097 & .075 & .036 & -.111 \\
Autoestima & -.042 & $.244^{* *}$ & -.001 & $-.148^{*}$ & -.098 & $.150^{*}$ \\
Complejidad & -.058 & $-.185^{* *}$ & .012 & $.329^{* *}$ & $.307^{* *}$ & -.026 \\
Sociabilidad & .082 & -.026 & .016 & -.073 & -.021 & .014 \\
Perfeccionismo & .049 & -.100 & .006 & .085 & -.094 & -.044 \\
Calma & .059 & .021 & .062 & .054 & -.003 & .040 \\
\hline
\end{tabular}

$* \mathrm{p}<.05 \quad * * \mathrm{p}<.01$

El análisis de la Tabla 3 permite concluir que los cinco factores medidos por el inventario se correlacionan significativamente con todas las escalas del CIEC, siendo Amabilidad y Cultura, las de mayor valor predictivo. Del análisis de las correlaciones factores primarios-escalas del CIEC, puede inferirse que ocho escalas primarias (Obediencia, Sensibilidad, Imaginación, Complejidad, Estabilidad, Autoestima, Calidez y Gregarismo) correlacionan significativamente con intención de elección de carreras. Las correlaciones más importantes fueron las verificadas entre las escalas Sensibilidad (.24), Imaginación (.25) y Complejidad (.30) con el factor carreras Artísticas, las escalas Sensibilidad (.33) y Complejidad (.33) con el factor carreras Humanistas, y las escalas Estabilidad (.23), Sensibilidad (-.43) y Autoestima (.24) con el factor carreras Tecnológicas.

En la actualidad se recomienda incluir procedimientos multivariados y no solamente bivariados para explicar el comportamiento humano (Tabachnik \& Fidell, 1989), en los estudios de validez predictiva, puesto que permiten esclarecer con mayor precisión la red de interrelaciones que se establecen entre grupos de predictores y un 
criterio (Thompson y Borrello, 1985). Por consiguiente, en una segunda fase de este estudio, se realizó un análisis de regresión múltiple (método Stepwise), para estimar el grado en que cada escala del 16PF IPIP contribuye, de manera independiente, a explicar el comportamiento de intenciones de elección de carrera. Las siguientes tablas presentan los resultados de este último estudio.

Tabla 5

Método stepwise para predecir intenciones de elección de carrera a partir de los Cinco Grandes Factores del 16PF-IPIP

\begin{tabular}{|c|c|c|c|c|}
\hline & Variable Dependiente & & & \\
\hline Predictor & & $\beta$ & $\mathrm{t}$ & $\mathrm{p}$ \\
\hline $\begin{array}{l}\text { Amabilidad } \\
\Delta \mathrm{R}^{2}\end{array}$ & $\begin{array}{c}\text { Carreras Científicas } \\
\begin{array}{c}(1,234) 7,546 * \\
.027\end{array}\end{array}$ & -.177 & $-2,747$ & .006 \\
\hline $\begin{array}{l}\text { Amabilidad } \\
\text { Responsabilidad } \\
\text { Cultura } \\
\Delta \mathrm{R}^{2} \quad \mathrm{~F}\end{array}$ & $\begin{array}{l}(3,233) 10,333^{* *} \\
.106\end{array}$ & $\begin{array}{l}.197 \\
-.201 \\
.150\end{array}$ & $\begin{array}{c}2,808 \\
-3,249 \\
2,151\end{array}$ & $\begin{array}{l}.005 \\
.001 \\
.033\end{array}$ \\
\hline $\begin{array}{l}\text { Amabilidad } \\
\Delta \mathrm{R}^{2}\end{array}$ & $\begin{array}{c}\text { Carreras Humanistas } \\
\begin{array}{c}(1.223) 31,494 * * \\
.119\end{array}\end{array}$ & .351 & 5,612 & .000 \\
\hline $\begin{array}{l}\text { Extraversión } \\
\Delta \mathrm{R}^{2} \quad \mathrm{~F}\end{array}$ & $\begin{array}{c}\text { Carreras Sociales } \\
\begin{array}{c}(1,231) 6,456^{*} \\
.023\end{array}\end{array}$ & .165 & 2,541 & .012 \\
\hline $\begin{array}{l}\text { Responsabilidad } \\
\Delta \mathrm{R}^{2}\end{array}$ & $\begin{array}{c}\text { Carreras Médicas } \\
\begin{array}{c}(1,230) 4,089 * \\
.013\end{array}\end{array}$ & .132 & 2,022 & .044 \\
\hline $\begin{array}{l}\text { Amabilidad } \\
\text { Estabilidad Emocional } \\
\qquad \mathrm{R}^{2} \quad \mathrm{~F}\end{array}$ & $\begin{array}{c}(2,228) 19,956 * * \\
.142\end{array}$ & $\begin{array}{l}-.347 \\
.149\end{array}$ & $\begin{array}{c}-5,664 \\
2,438\end{array}$ & $\begin{array}{l}.000 \\
.016\end{array}$ \\
\hline
\end{tabular}




\section{Tabla 6}

Método stepwise para predecir intenciones de elección de carrera a partir de la 16 escalas primarias del 16PF IPIP.

\begin{tabular}{|c|c|c|c|c|}
\hline \multirow[b]{2}{*}{ Predictor } & \multirow{2}{*}{ Variable Dependiente } & \multirow[b]{2}{*}{$\beta$} & \multirow[b]{2}{*}{$\mathrm{t}$} & \multirow[b]{2}{*}{$\mathrm{p}$} \\
\hline & & & & \\
\hline & Carreras Científicas & & & \\
\hline Sensibilidad & & -.146 & $-3,07$ & .024 \\
\hline $\mathrm{F}$ & $(1,234) 9,469 * *$ & & & \\
\hline \multirow[t]{2}{*}{$\Delta \mathrm{R}^{2}$} & .017 & & & \\
\hline & Carreras Artísticas & & & \\
\hline Complejidad & & .254 & 3,47 & .001 \\
\hline Obediencia & & -.152 & $-2,50$ & .013 \\
\hline Asertividad & & -.166 & $-2,57$ & .011 \\
\hline Gregarismo & & .178 & 2,84 & .005 \\
\hline Sensibilidad & & .149 & 2,11 & .035 \\
\hline $\mathrm{F}$ & $(5,231) 9,841 * *$ & & & \\
\hline \multirow[t]{2}{*}{$\Delta \mathrm{R}^{2}$} & .158 & & & \\
\hline & Carreras Humanistas & & & \\
\hline Sensibilidad & & .220 & 3,09 & .003 \\
\hline Complejidad & & .209 & 2,79 & .005 \\
\hline $\mathrm{F}$ & $(2,222) 17.896^{* *}$ & & & \\
\hline \multirow[t]{2}{*}{$\Delta \mathrm{R}^{2}$} & .131 & & & \\
\hline & Carreras Sociales & & & \\
\hline Calidez & & .191 & 2.81 & .003 \\
\hline $\mathrm{F}$ & $(1,231) 8.777^{* *}$ & & & \\
\hline \multirow[t]{2}{*}{$\Delta \mathrm{R}^{2}$} & .032 & & & \\
\hline & Carreras Médicas & & & \\
\hline Obediencia & & .178 & 2.81 & .006 \\
\hline $\mathrm{F}$ & $(1,231) 7.910^{*}$ & & & \\
\hline \multirow[t]{2}{*}{$\Delta \mathrm{R}^{2}$} & .028 & & & \\
\hline & Carreras Tecnológicas & & & \\
\hline Sensibilidad & & -.362 & $-6,05$ & .000 \\
\hline Estabilidad & & .161 & 2,67 & .008 \\
\hline Gregarismo & & .265 & 3,53 & .000 \\
\hline Amigabilidad & & -.169 & $-2,31$ & .022 \\
\hline Sociabilidad & & -.121 & $-1,981$ & .049 \\
\hline $\mathrm{F}$ & $(5,225) 14.269 * *$ & & & \\
\hline$\Delta \mathrm{R}^{2}$ & .224 & & & \\
\hline
\end{tabular}


El primer análisis (tabla 5) permite corroborar la utilidad predictiva de los cinco factores con relación a intenciones de elección de carrera. Amabilidad parece ser el factor con mayor poder explicativo, los demás factores explican porcentajes muy reducidos de la varianza de "intención de elección de carreras". Por último, el análisis de la contribución explicativa de las facetas específicas permite inferir las siguientes conclusiones.

En el primer modelo de regresión la variable dependiente fue el factor de carreras "Científicas", y la variable independiente Sensibilidad explicó un $2 \%$ de la varianza, $F(1,234)=9,469, p<.002$. La única escala incluida en el análisis fue Sensibilidad, que contribuye de forma independiente con un valor de $\beta .146, t=-3,07$, $p<.002$.

En el segundo modelo, la variable dependiente fue el factor carreras "Artísticas", y las variables independientes incluídas en el análisis fueron las escalas Complejidad, Obediencia, Asertividad, Gregarismo y Sensibilidad, que explican en conjunto un $18 \%$ de la varianza, $\mathrm{F}(5,231)=9,841, p<.000$. Asimismo, cabe señalar la contribución explicativa independiente que realizan cada una de las escalas: Complejidad ( $\beta .254, t=$ $3,47, p<.001)$, Gregarismo $(\beta .178, \boldsymbol{t}=2,84, p<.005)$, Sensibilidad $(\beta .149, \boldsymbol{t}(240)=$ $2,11, p<.035)$, Obediencia $(\beta .152, \boldsymbol{t}=-2,50, p<.013)$, y Asertividad $(\beta .166, \boldsymbol{t}=-2,57$, $p<.011)$.

En el tercer modelo, la variable dependiente fue el factor carreras "Humanistas", y las variables independientes incluídas en el análisis fueron las escalas Sensibilidad, $(\beta$ $.220, \boldsymbol{t}=3,09, p<.002)$ y Complejidad $(\beta .209, \boldsymbol{t}=2,79, p<.006)$, explicando en conjunto un $14 \%$ de la varianza, $\mathrm{F}(2,222)=17.896, p<.000$.

En el cuarto modelo, la variable dependiente fue carreras "Sociales" y la variable independiente Calidez, $\beta .191, t=2.81, p<.005$ explicó un $4 \%$ de la varianza, $\mathrm{F}$ $(1,231)=8.777, p<.003$.

En el quinto modelo, la variable dependiente fue el factor de carreras "Médicas", y la única variable independiente que contribuyó fue la escala Obediencia, $\beta$ (.178), $t$ $(240)=2.81, p<.005$, explicando un $3 \%$ de la varianza, $\mathrm{F}(1,231)=7.910, p<.005$.

En el último modelo, la variable dependiente fue el factor carreras "Tecnológicas", y el conjunto de variables independientes aportan la mayor contribución explicativa, $24 \%$ de intenciones de elección de carrera, $F(5,225)=$ 
14.269, $p<.000$. Las escalas significativamente predictivas fueron Sensibilidad ( $\beta$ $.362, \boldsymbol{t}=-6,05, p<.000)$, Amigabilidad $(\beta-.169, \boldsymbol{t}=-2,31, p<.022)$, Sociabilidad $(\beta-$ $.121, \boldsymbol{t}=-1,981, p<.049)$, Estabilidad $(\beta .161, t=2,67, p<.008)$, y Gregarismo ( $\beta .265$, $\boldsymbol{t}=3,53, p<.000)$.

De acuerdo a estos resultados, la escala Sensibilidad parece ser la de mayor poder explicativo, evidenciando utilidad predictiva con relación a tres de las escalas del CIEC (Carreras Científicas, Humanistas y Tecnológicas). La contribución de los factores y facetas del 16PF IPIP para explicar las intenciones de elección de carreras "Sociales" y "Médicas" son muy modestas.

\section{Discusión}

En los últimos años, en Orientación de Carrera, ha resurgido el interés por la medición de la personalidad. Se han realizado estudios intentando vincular modelos teóricos de la personalidad con otros de los intereses vocacionales y determinar el papel de la evaluación de personalidad en orientación vocacional. El modelo de los cincos factores suministra una descripción razonablemente abarcativa y un avance en la taxonomía del anteriormente anárquico campo de la evaluación de la personalidad. Pese a las críticas respecto a que este modelo carece de una teoría biológica o psicológica explícita de la personalidad, en los últimos años se han recolectado varias evidencias empíricas que lo ligan a las bases genotípicas de la personalidad y los mecanismos funcionales del cerebro (Loehlin et al, 1998).

En la actualidad se cuenta con dos modelos de los cincos factores, uno desarrollado por Costa \& MaCrae (1992), y el otro asociado con los estudios basados sobre la hipótesis léxica (Goldberg, 1999), que discrepan ligeramente entre sí. Existen una gran variedad de instrumentos basados en este modelo, aunque la mayoría son propiedad de sus autores $\mathrm{y}$, como consecuencia de esto, es dificultoso para otros científicos contribuir a su desarrollo posterior y refinamiento. Frente a esta problemática Lewis Goldberg (1999) ha propuesto un sitio en Internet (http://ipip.ori.org/ipip/), el Pool Internacional de Ítems de Personalidad (IPIP, por sus siglas en inglés), donde las escalas IPIP del 16PF han evidenciado las mejores propiedades psicométricas. Por consiguiente, nos propusimos traducir y adaptar el Inventario de Personalidad 16PF-IPIP. 
Se han realizados tres estudios conducentes a la adaptación del Inventario de Personalidad 16PF-IPIP de Goldberg para evaluación de adolescentes de nuestro medio que desean ingresar a carreras de nivel superior.

El estudio comparativo entre la versión original y la traducida del inventario de personalidad, demostró que ambos instrumentos pueden ser considerados equivalentes. No obstante, sería necesario aplicar procedimientos de Funcionamiento Diferencial de los Items (Nunnally \& Bernstein, 1995) para refinar la información obtenida y determinar con precisión aquellos ítems que no se presenten como estrictamente equivalentes. La consistencia interna de casi todas las escalas es adecuada (sólo la escala calidez presenta un coeficiente alfa moderado), y se obtuvo un coeficiente alfa promedio en la versión en Español casi similar a la original en inglés (versión española .77 y versión original .80).

Los estudios de validez de criterio, realizados en dos fases, demuestran la utilidad predictiva de algunas escalas con relación a intenciones de elección de carrera. En una primera etapa se realizó un estudio bivariado entre las 16 escalas del 16PF IPIP y los seis factores de intenciones de elección de carrera medidos por el CIEC. Amabilidad es el único factor con un contribución interesante para la explicación de intención de elección de carreras. Para poder estimar con mayor precisión cual es la contribución explicativa de cada faceta del inventario de personalidad de manera independiente, realizamos, en una segunda fase, un estudio de análisis de regresión múltiple, aplicando el método Stepwise. Tanto en los análisis bi como multivariados, se observa una moderada utilidad predictiva de algunas escalas del inventario de personalidad con relación a intenciones de elección de carrera. Sensibilidad y Complejidad (facetas de los factores Amabilidad y Cultura, respectivamente), en ese orden, parecen ser variables promisorias que podrían incrementar la información explicativa que, tradicionalmente, aportan los inventarios de intereses y de autoeficacia respecto a intención de carreras (Lent, Brown \& Hackett, 1994; Fogliatto \& Pérez, 2003). Coincidentemente con la literatura (Costa \& McCrae, 1999), las facetas de los factores Estabilidad, Responsabilidad y Extraversión, parecen tener poco peso a la hora de explicar el comportamiento de elección de carrera, y en otros estudios futuros debería verificarse su utilidad para otras dimensiones del comportamiento de carrera (Rendimiento Académico, por ejemplo). 
En general, los resultados alcanzados son alentadores para algunas escalas, aunque es necesario planificar nuevos estudios que comparen la utilidad predictiva de diferentes variables críticas (intereses, habilidades objetivas, autoeficacia, rasgos de personalidad) en un contexto de orientación. Estas investigaciones permitirían esclarecer la validez incremental aportada por cada uno de estos factores psicológicos, para la explicación de variables importantes en el desarrollo de carrera, tales como rendimiento académico, elección de carrera y satisfacción. En ese sentido, un estudio reciente realizado paralelamente a éste, ha verificado la utilidad predictiva del Factor Responsabilidad y la faceta Perfeccionismo con relación a rendimiento académico (medido por el promedio general de calificaciones en todas las asignaturas) en estudiantes de último año de Polimodal (Ayllón, 2004), con una contribución independiente a la realizada por Habilidad Cognitiva General (medida por el Test de Aptitudes Diferenciales, subtests de Razonamiento Verbal y Numérico) y Autoeficacia para Inteligencias Múltiples (medida por el Inventario de Autoeficacia para Inteligencias Múltiples, escalas Lingüística y Lógico-Matemática).

Nuestra intención es construir un inventario breve de rasgos de personalidad con implicancia vocacional, seleccionando aquellas escalas del 16PF-IPIP que evidencien mayor utilidad predictiva, a través de una serie de estudios pertinentes que se planifican. Este procedimiento, de selección de las escalas más relevantes del FFM en un ámbito específico, ya se ha realizado exitosamente (Gustavsson, et al., 2002) en el ámbito de la evaluación de rasgos de personalidad relevantes para la salud, mediante la construcción de un inventario de facetas específicas del FFM relevantes en el ámbito de la salud (Health Personality Inventory, HPI - 5).

\section{Referencias}

Allport, G.W. and Odbert, H.S. (1936). Trait-names: A psycho-lexical study. Psychological Monographs, 47, 1, 211.

Ayllón, S. (2004). Un modelo social-cognitivo del rendimiento académico de estudiantes de Polimodal. Tesis de Maestría. Inédita.

Block, J. (1995). A contrarian view of the five-factor approach to personality description. Psychological Bulletin, 117,185-215. 
Block, J. (2001). Mellennial Contrarianism: The Five-Factor aproach to personality description 5 years later. J. Res. Personal. 35, 98-107.

Bouchard, T. J. and Loehlin, J.C., (2001). Genes, Evolution, and Personality. Behavior Genetics, 31, 3, 244-273.

Cattell, R.B. (1947). Confirmation and clarification of primary personality factors. Psychometrika, 12, 197-220.

Cloninger, C (1988). A unified biosocial theory of personality and its role in the development of anxiety states: A reply to commentaries. Psychiatric Developments, 2, 83-120.

Costa, Jr., P.T and McCrae, R.R. (1992) Four ways five factors are basic. Personality and Individual Differences, 13, 653-665.

Costa, P \& McCrae, R. (1999) Inventario de Personalidad NEO Revisado. Madrid: TEA.

Digman, J.M. and Takemoto-Chock, N.K. (1981). Factors in the natural language of personality: Re-analysis, comparison, and interpretation of six major studies. Multivariate Behavioral Research, 16, 149-170.

Digman, J.M., (1990). Personality structure: Emergence of the five-factor model. In: M.R. Rosenzweig and L.W. Porter, Editors, Annual review of psychology, 41, 417-440.

Eysenck, H.J. (1991) Dimensions of personality: 16, 5, or 3? - Criteria for a taxonomic paradigm. Personality and Individual Differences, 12, 773-790.

Eysenck, H.J. (1992). Four ways five factors are not basic. Personal Indiv. Diff. 13, 667-673.

Fogliatto, H. (1991). Cuestionario de intereses profesionales. Manual. Buenos Aires: Guadalupe.

Fogliatto, H. \& Pérez, E. (2003). SOVI 3. Manual y CD-ROM. Buenos Aires: Paidós

Galton, F.,(1884). Measurement of character. Fortnightly Review 36, 179-185.

Goldberg, L.R., (1992). The development of markers of the Big-Five factor structure. Psychological Assessment, 4, 26-42.

Goldberg, (1993).The Structure of Phenotypic Personality Traits. American Psychological Association, 48, 26-34. 
Goldberg, L.R., (1999) A broad-bandwidth, public-domain, personality inventory measuring the lower-level facets of several Five-Factor models, en Mervielde y Col. Personality Psychology in Europe, 7.

Goldberg, L.R., (2001). The Comparative Validity of Adult Personality Inventories: Applications of a Consumer-Testing Framework. In S. R. Briggs, J. M. Cheek, \& E. M. Donahue (Eds.). Handbook of Adult Personality Inventories. New York: Plenum Publishing Corp

Gottfreson, G; Jones, E \& Holland, J (1993) Personality and Vocational Interest: The relation of Holland's six interest dimensions to five robust dimensions of Personality. Journal of Counseling Psychology, 40, 518-524.

Gustavsson, J. P.; E. G.; Linder, J. \& Robert M. Weinryb R. M. (2002). The HP5 inventory: definition and assessment of five health-relevant personality traits from a five-factor model perspective. Personality and Individual Differences. 35, 1, 6989.

Holland, J (1997). Self-Directed Search. Technical Manual. Odessa: Psychological Assessment Resources.

Jang, K. L., Livesley, W. J., and Veron, P. A (1996). Heritability of the big five personality dimensions and their facets. A twin study. Journal of Personality, 64, 438-444.

John, O.P.,(1990). The "Big Five" factor taxonomy: Dimensions of personality in the natural language and in questionnaires. In: L.A. Pervin, Editor, Handbook of personality: Theory and research, Guilford Press, 66-100.

Johnson, D.L., Wiebe, J.S., Gold, S.M., Andreasen, N.C., Hichwa, R.D., Watkins, G.L. and Ponto, L.L.B. (1999) , Cerebral blood flow and personality: A positron emission tomography study. American Journal of Psychiatry, 156, 252-257.

Larson, L.M., Rpttinghaus, P.J. and Borgen, F. H.(2002). Meta-analyses of Big Six Interests and Big Five personality Factors. Journal of Vocational Behavior, 61, 217-239.

Lent, R.; Brown, S., \& Hackett, G. (1994). Toward a unifying social cognitive theory of career and academic interest: Choice and performance. Journal of Vocational Behavior, 45, $79-122$. 
Loehlin, J.C., McCrae, R. R., Costa, P. T. Jr. \& John, O. P. (1998). Heritabilities of Common and Measure-Specific Components of the Bbig Five Personality Factors. Journal of Research in Personality, 32, 432-453.

Lowman, R (1991) The clinical practice of carrer assessment: interest, abilities and personality. Washington: American Psychological Asociation.

MacAdams, D.P. (1992). The five-factor model in personality: A critical appraisal. Journal of Personality, 60, 329-361.

McCrae, R.R. and Costa Jr., P.T. (1992). Discriminant validity of NEO-PIR facet scales. Educational and Psychological Measurement, 52, 229-237.

McCrae, R. R. \& Costa, P. T., Jr. (1999) A five- factor theory of personality. In L. Pervin \& O. P. John (Eds). Handbook of personality. (2 ${ }^{\text {nd }}$. Ed., pp. 139 - 156). New York: Guilford Press.

McCrae, R. R. (2003). Human nature and culture: A trait perpective. Journal of Research in Personality, 38, 3-34.

Muñiz, J. (2001). Teoría clásica de los tests. Madrid, Síntesis Psicológica.

Norman, W.T. (1963). Toward an adequate taxonomy of personality attributes. Replicated factor structures in peer nomination personality ratings. Journal of Abnormal and Social Psychology, 66, 574-583.

Nunnally \& Bernstein. (1995). Teoria Psicométrica. Mexico: McGraw Hill.

Pérez, E. (2001). Construcción de un Inventario de Autoeficacia para Inteligencias Múltiples. Tesis Doctoral. Inédita. Facultad de Psicología. Universidad Nacional de Córdoba.

Pérez, E. \& Baldo, M. (2002). Construcción y Adaptación de Tests Psicológicos. Nuevos Desarrollos en la Ciudad de Córdoba. Córdoba: Ed. Brujas.

Rewelle, W. (1995). Personality Processes. Annual Review of Psychology, 46, 295- 328.

Riemann, R., Angleitner, A. \& Strelau, J.(1997). Genetic and environmental influences on personality: A study of twins reared together using the self-and peer report NEO-FFI scale. Journal of Personality, 65, 449-475.

Russell, M \& Carol, D. (2000) 16PF 5 Manual.Madrid: TEA.

Saucier, G. and Goldberg, L. R. (1995) So What Do You Propose We Use Instead? A Reply to Block1, Psychological Bulletin, 117, 2, 221-225 
Stuogh, C., Donaldson, C., Scarlata, B., \& Ciorciari, J, (2001). Psychophysiological correlates of the NEO PI-R Openness, Agreeableness and Conscientiousness: preliminary results. International Journal of Psychophysiology, 41, 1, 87-91.

Tabachnik, B. \& Fidell, L. (1989). Using multivariate statistics. New York: Harper \& Row.

Thompson, B. \& Borrello, G. (1985). The importance of structure coefficients in regresion research. Educational and Psychological Measurement. 203 - 209.

Tokar, D. M., Fischer, A. R., \& Subich, L. M. (1998). Personality and Vocational behavior: A selective review of the literature, 1993-1997. Journal of Vocational Behavior, 53, 115-153.

Tupes, E.C. and Christal, R.E. (1961). Christal Recurrent personality factors based on trait ratings (USAF ASD Tech. Rep., 61-97), U.S. Air Force, Lackland Air Force Base, TX.

Tupes, E.C. and Christal, R.E., (1992). Recurrent personality factors based on trait ratings. Journal of Personality, 60, 225-251.

Waller, N. G. (1999). Evaluating the structure of personality. In Cloninger, C. R. (ed), Personality and Psychopathology, American Psychiatric Association, 155-197. 


\section{APÉNDICE}

\section{ÍTEMS DEL INVENTARIO 16PF-IPIP. VERSIÓN PRELIMINAR EN ESPAÑOL}

\section{Calidez}

1 Sé como alentar a los demás.

Me agrada recibir personas.

Me emocionan los sentimientos de los otros.

Me intereso por la vida de otras personas.

Alegro a las demás personas.

Hago sentir cómoda a la gente.

Dedico tiempo a otras personas.

No me gusta involucrarme en los problemas de los demás.

No me intereso demasiado por los demás.

10 Trato de no pensar en las personas necesitadas.

\section{Intelecto}

11 Hago observaciones claras.

Conozco las respuestas para muchos interrogantes.

Tiendo a analizar todas las cosas.

Empleo mucho el razonamiento.

Aprendo rápidamente.

Soy crítico con los argumentos de los demás

Reflexiono antes de actuar.

Analizo los pros y los contras de cada situación.

Me considero una persona común

Me confundo con facilidad

Sé que no soy una persona muy destacada .

Tengo un vocabulario limitado.

23 Cuando estoy leyendo paso por alto las palabras difíciles.

\section{Estabilidad}

24 Pocas veces me siento triste.

Me siento cómodo conmigo mismo.

Supero fácilmente las dificultades.

Estoy tranquilo la mayor parte del tiempo.

No me siento frustrado fácilmente.

Tengo cambios frecuentes en mi estado de ánimo.

Frecuentemente me siento triste.

No me agradan muchos aspectos de mí mismo.

Frecuentemente siento desesperación.

$33 \mathrm{Me}$ desaliento con facilidad.

\section{Asertividad}

34 Frecuentemente asumo responsabilidades.

Me agrada hacerme responsable 
Generalmente digo lo que pienso.

No siento temor de expresar mis críticas.

Tengo todas las cosas controladas

Puedo tomar medidas rigurosas con los demás.

Casi siempre espero que los demás tomen la iniciativa.

Casi nunca critico a los demás.

42 Dejo que los demás tomen las decisiones.

\section{Gregarismo}

43 Me dejo conducir por los demás.

Soy el alma de las fiestas.

Me agradan las fiestas prolongadas.

Hago muchas bromas.

Disfruto entre la multitud.

Divierto a mis amigos.

Frecuentemente actúo de manera impulsiva.

Casi nunca hago bromas.

No me agradan las multitudes.

Soy el último en festejar una broma.

53 No me gusta escuchar música con volumen alto.

\section{Obediencia}

54 Creo que las leyes deben cumplirse estrictamente.

Intento respetar las leyes.

Creo en una única religión verdadera.

Respeto la autoridad.

Me gusta ponerme de pie durante el himno nacional.

Me resisto a la autoridad.

Desobedezco las leyes

Frecuentemente utilizo malas palabras.

Me opongo a la autoridad.

63 Sé como evadir las leyes.

\section{Amigabilidad}

64 Me siento cómodo entre la gente.

En las fiestas hablo con diferentes tipos de personas.

No me importa ser el centro de la atención de los demás.

Hago amigos con facilidad.

Generalmente soy yo quien comienza las conversaciones.

Encuentro difícil acercarme a los demás.

Frecuentemente me siento incómodo entre los demás.

Generalmente no tengo mucho para decir.

Cuando estoy entre desconocidos me mantengo callado.

73 Intento no llamar la atención.

\section{Sensibilidad}

74 Me gusta leer

Disfruto las discusiones sobre literatura. 
Leo mucho

No me gustan las películas de acción.

Lloro frecuentemente durante las películas.

Adoro las flores

No disfruto los espectáculos de danza.

No me agrada la poesía.

82 No me agrada la literatura de ficción (novelas, por ejemplo)

\section{Confianza}

83 Casi nunca soy conciente de mis reacciones emocionales.

Me resulta difícil perdonar a los demás.

Creo que los demás tienen malas intenciones.

Soy precavido con los demás.

Desconfío de la gente.

Creo que las personas pocas veces dicen toda la verdad

Creo que las personas son malas por naturaleza.

Confío en lo que dicen las otras personas.

Confío en los otros

Creo que los demás tienen buenas intenciones.

93 Creo que las personas son honestas por lo general.

\section{Imaginación}

94 Hago cosas que a los demás les resultan extrañas.

Me gusta sumergirme en mis pensamientos.

Disfruto dejando volar mi imaginación.

Adoro soñar despierto.

Siempre voy contra la corriente.

Tengo opiniones diferentes del resto de la gente.

Hago cosas inesperadas.

Hago las cosas de manera estructurada.

Casi nunca sueño despierto.

103 Pocas veces me distraigo en mis pensamientos.

\section{Apertura}

104 Revelo poco de mí mismo.

A los demás les resulta difícil conocerme.

No hablo mucho.

Escondo mis sentimientos.

Soy reservado con mis pensamientos.

Soy abierto con los demás respecto a mí mismo

Soy abierto respecto a mis sentimientos.

Comunico mis pensamientos íntimos.

Muestro mis sentimientos.

113 Siento deseos de hablar de mí mismo.

\section{Autoestima}

114 Tengo miedo de equivocarme.

Con frecuencia me siento presionado 
Me hieren con facilidad.

Me preocupo demasiado.

Pienso mucho en mis errores pasados.

Siento culpa cuando digo "no".

Generalmente los problemas me abruman.

No me preocupan las cosas que ya han ocurrido.

No me altero con facilidad.

123 No me dejo desalentar por lo demás.

\section{Complejidad}

$124 \mathrm{El}$ arte es muy importante para mí

Me gusta imaginar nuevas formas de hacer las cosas.

Disfruto escuchando nuevas ideas

Trato de elevar el nivel intelectual de las conversaciones.

Me gusta más la variedad que la rutina.

Evito las discusiones filosóficas.

Casi nunca investigo el significado profundo de las cosas.

No me interesan las discusiones teóricas.

No me interesan las ideas abstractas.

133 Trato de evitar a las personas complejas

\section{Sociabilidad}

134 Me gusta estar solo.

Prefiero hacer las cosas yo solo.

Disfruto los momentos de soledad.

Busco la tranquilidad.

No me molesta comer solo.

Disfruto el silencio.

Disfruto de mi privacidad.

Disfruto participando en un grupo.

Disfruto trabajando en grupo.

143 No puedo hacer nada sin la compañía de los demás.

\section{Perfeccionismo}

144 Trato de hacer todas las cosas correctamente

Realizo mis tareas inmediatamente.

Me agrada el orden.

Persevero hasta que todo esté perfecto.

Soy muy preciso en mi trabajo.

No me molesta la gente desordenada.

No me molesta el desorden.

Mi habitación está siempre revuelta.

Dejo mis cosas en cualquier lado.

153 Postergo las tareas desagradables.

\section{Calma}

$154 \mathrm{Me}$ irrito con facilidad.

Me enojo fácilmente 
Soy rápido para juzgar a los demás.

Me molestan los errores de la gente.

Me descontrolo con facilidad.

No puedo tolerar que me contradigan.

La apariencia de los demás es muy importante para mí.

No me enojo fácilmente.

Intento olvidar y perdonar.

163 Siempre tengo una palabra de aliento para todos. 